\title{
ZU HAUSE IN DER DEUTSCHEN SPRACHE: ZWEISPRACHIGKEIT UND SPRACHMISCHUNGEN IN DEN TEXTEN VON RADEK KNAPP
}

\begin{abstract}
Die Schriftsteller mit dem sog. Migrationshintergrund gehören zu denjenigen Menschen, die als Migranten einen einschneidenden Kultur- und auch Sprachwechsel hinter sich haben und deren Lebenslauf durch diesen Wechsel zwischen Sprachen und Lebensformen geprägt ist. Die neue Sprache ermöglicht den Zugang zu einer neuen, anderen Kultur, somit auch den Zugang zu einer neuen kulturellen Identität sowie - last but not least - zu einem neuen Lesepublikum, was im Falle des literarischen Schaffens doch von entscheidender Bedeutung sein kann, weil die Schriftsteller sich absichtlich für einen neuen Adressatenkreis entscheiden. Obwohl man meist in der Sprache den wichtigsten Identitätsmarker sieht und anerkennt, begründet das Deutsche als literarisches Ausdrucks- und Publikationsmedium im Falle der transkulturellen Schriftsteller keine homogenen nationalen Identitäten, sondern erfüllt die Funktion eines Transitraumes, einer-zone, eines transkulturellen Raumes der gegenseitigen Durchdringung und Verflechtung des Kulturellen sowie der Transformation nationaler Zugehörigkeiten und der kulturellen Identität. In den Texten der Migrantenautoren spiegelt sich die Bedeutung des Umgangs mit zwei Sprachen wider, weil die Zweisprachigkeit von Migranten - oft im Zusammenhang mit dem Thema der kulturellen Identität - in der Migrantenliteratur eine Rolle spielt. Die Schriftsteller versuchen, ihre eigene Zweisprachigkeit in die Texte einzubringen, in denen häufig beide Kulturen und Sprachen koexistieren. Im Referat werden ausgewählte Texte von dem polnisch-österreichischen Schriftsteller Radek Knapp einer Analyse unterzogen. Der Beitrag geht der Frage nach, wo und wie sich die Zweisprachigkeit und Sprachmischungen in den einzelnen Texten Knapps manifestieren.
\end{abstract}

SCHLÜSSELWÖRTER: österreichisch-polnische Literaturbeziehungen, österreichische Gegenwartsliteratur, kulturelle Identität, Migrantenliteratur, Zweisprachigkeit, Schriftsteller mit dem Migrationshintergrund, Radek Knapp

\section{AT HOME IN THE GERMAN LANGUAGE: BILINGUALISM AND LANGUAGE MIX- TURES IN THE TEXTS OF RADEK KNAPP}

ABSTRACT: The writers with the so-called migration background are among those people who have undergone a dramatic change of culture and language as migrants and whose curriculum vitae is shaped by this change between languages and ways of life. The new language allows access to a new, different culture, thus also access to a new cultural identity and - last but not least - to a new reading public,

Agnieszka Palej - Uniwersytet Jagielloński, Kraków, agnieszka.palej@uj.edu.pl 
which in the case of literary creation can be of crucial importance because the writers deliberately decide on a new addressee group. Although one mostly sees and acknowledges the most important identity marker in language, German as a literary medium of expression and publication in the case of transcultural writers does not establish homogeneous national identities, but fulfills the function of a transit space, a zone, a transcultural space of interpenetration and interweaving the cultural as well as the transformation of national affiliations and cultural identity. The texts of migrant authors reflect the importance of dealing with two languages because the bilingualism of migrants, often in the context of cultural identity, plays a role in migrant literature. The writers try to bring their own bilingualism into the texts, where often both cultures and languages co-exist. In the presentation selected texts are subjected to an analysis by the Polish-Austrian writer Radek Knapp. The article explores the question of where and how bilingualism and language mixtures manifest themselves in the individual texts of Knapp.

KEYWORDS: Austrian-Polish literary relationships, Austrian contemporary literature, cultural identity, bilingualism, authors with a migration background, Radek Knapp

Die Autoren mit dem sog. Migrationshintergrund haben einen einschneidenden Kultur- und meistens auch Sprachwechsel hinter sich. Die Tatsache, dass sie nicht nur in zwei Kulturen, sondern auch in mindestens zwei Sprachen leben, beeinflusst natürlicherweise die literarischen Texte dieser Schriftsteller. Unter Sprachwechsel, einem „der irritierendsten literarischen Phänomene der Moderne“ (Lamping 1996: 33), wird der Wechsel eines Individuums von einer Sprache zu einer anderen verstanden. In den meisten Fällen ist es eine freie, subjektiv vollzogene Entscheidung des Einzelnen, eine andere Sprache zum künstlerischen Ausdrucksmittel zu machen (vgl. Trepte 2000: 247) und kann als ein Merkmal der Moderne bzw. Postmoderne aufgefasst werden. Die Aufhebung der kulturellen Grenzen in den Zeiten der Globalisierung kann auch auf die Sprache bezogen werden. Saša Stanišić, selbst ein deutschsprachiger Autor mit Migrationshintergrund, macht auf die Tatsache aufmerksam, dass die Sprache „das einzige Land ohne Grenzen“ sei (Stanišić 2008: 109). Wenn man seine Sprache wechselt, bildet diese Entscheidung aber eine markante Zäsur sowohl im Privatleben als auch - im Falle der Schriftsteller - in ihrem literarischen Schaffen. Wichtig ist es zu unterscheiden, ob es sich dabei um einen freien Entschluss handelt, oder ob diese Entscheidung durch die Lebensumstände sozusagen erzwungen worden ist. Eine wichtige Rolle kann dabei selbstverständlich auch die sprachlich-kulturelle Anziehungskraft der neuen Heimat spielen.

Die neue Sprache ermöglicht den Zugang zu einer neuen, anderen, unbekannten Kultur und vielleicht infolgedessen auch zu einer neuen kulturellen Identität. Der Sprachwechsel ermöglicht außerdem eine gewisse Distanz zum Heimatland, seiner kulturellen Tradition und auch der Muttersprache sowie einen bewussten Umgang mit der Sprache überhaupt. Der Sprachwechsler trennt sich von den Traditionen seiner Muttersprache und gleichzeitig von sprachlichen und kollektiven Tabus und Mythen, Normen und Verhaltensmustern. Diese Trennung kann auch als eine Art Befreiung empfunden werden. Durch den Sprachwechsel schreiben die Migrantenautoren absicht- 
lich für einen anderen Adressatenkreis und finden Eingang in einen anderen Sprachund Kulturraum. Heute, im zusammenwachsenden, multi-, inter- und transkulturell geprägten Europa scheinen der Sprachwechsel und die doppelte Sprachbürgerschaft immer selbstverständlicher zu werden. Vor dem Hintergrund der veränderten Kulturkonzepte muss auch die Kategorie der kulturellen Identität neu reflektiert werden. Es kommt, worauf Hans-Christian Trepte aufmerksam macht, zu einer Hinterfragung und gleichzeitigen Neubewertung ethnischer und kultureller Identität, wobei Begriffe wie Heimat und Nation oft nicht länger stabile Kategorien darstellen (vgl. Trepte 2000: 248). Die Migrantenautoren kann man meines Erachtens nicht unbedingt als die zu der jeweiligen Nationalliteratur und -kultur Zugehörigen betrachten. Sie scheinen eine Sonderkategorie zu bilden und im Transkulturellen situiert zu sein. Hans-Christian Trepte macht einen Begriffsvorschlag. Trepte schlägt nämlich - in Anlehnung an die tschechische Exilschriftstellerin Vera Linhartová - den Begriff des „Nomaden“ (nomads) vor: Der Nomade wird in der zeitgenössischen Literatur oft zum Inbegriff von Beweglichkeit und Freiheit, er steht für das Wandern zwischen den Welten, für den Willen, den vertrauten Raum (seine Heimat, den vertrauten Kulturkontext) zu verlassen und Neues, Unbekanntes zu wagen. Der Nomade ist zu einer positiv besetzten Figur in den gegenwärtigen globalisierten Gesellschaften geworden, weil sie von den Individuen Mobilität und Flexibilität fordert. Der migrierende Autor wird als Nomade (Transmigrant) wahrgenommen, der eine ,nomadische“ Begegnung mit dem Anderen sowie dem Anderssein wagt. Die „Sesshaften“ bleiben ihrer Muttersprache im Gastland treu, isolieren sich gewollt (absichtlich) von der neuen kulturellen Umgebung, für sie besteht somit kein Identitätsproblem. Für die ,Nomaden“ kann jeder Ort, den sie betreten, zur Heimat werden. Die Heimat als ,kein Ort nirgends“ (quasi als Niemandsland), als ein Zustand des Unbehaustseins kann „mit der freien Wahl der Sprache einhergehen“ (Trepte 2000: 255, vgl. Linhartová 1997). Hans-Christian Trepte ist zu Recht davon überzeugt, dass das nomadisierende Unterwegssein ,im Dschungel der Weltmetropolen“ den Blick des „fremden Ankömmlings frisch“ hält und eine schöpferische Herausforderung darstellt: „Sich dem Leben in der Fremde zu stellen, wird so mit einem erneuten ,Auf-die-Welt-Kommen' verglichen, der eigentlich ein Akt des ,In-die-Welt-Kommens darstellt, der einer zweiten Geburt, einem zweiten Leben gleicht“" (Trepte 2000: 255). Die „Schriftstellernomaden“ betrachten die eigene Kultur mit anderen Augen und lernen, die ,erstaunlichen Möglichkeiten eines ,Zu-Hause-Sein im Nicht-Zuhause ${ }^{6}$ zu schätzen. Sie sind überall zu Hause, gleich einer Schnecke, die - wohin sie sich auch bewegt - ihr Schneckenhäuschen mit sich trägt" (Trepte 2000: 255, vgl. auch Trepte 2013: 92). Die Vergangenheit wird zur Erinnerung, die sie auf den Weg mitnehmen und mit der sie als Künstler kreativ umgehen. Die „Schriftstellernomaden“ passieren Grenzen, bewegen sich im Grenzgebiet und sind in der Lage oder werden vielleicht durch die neuen Schaffens- und Lebensumstände dazu gezwungen, ihre kulturelle Identität neu zu formulieren bzw. eine Wahl zu treffen. Manche identifizieren sich als bi- oder sogar plurikulturelle Personen. 
Die Folgen des Wechsels können positiver und negativer Natur sein. Eine positive Wirkung für den Schriftsteller ist seine doppelte Sprachbürgerschaft mit ihrer permanenten Auseinandersetzung mit beiden Sprachen (mit beiden Sprachkodes) sowie die potentielle Eroberung einer neuen, fremdsprachigen Leserschaft, der Gewinn eines neuen Adressatenkreises, Überwindung der Fremdheit, in vielen Fällen sogar der schriftstellerische Erfolg und Anerkennung in dem neuen Kulturkreis, als negativ können ein mögliches Gefühl der Zerrissenheit, die Gefahr des Sprachverlustes, der Verlust (das Ausbleiben) von traditionellen sprachlichen und kulturellen Bindungen, sogar die Entwurzelung betrachtet werden. Die Wahl einer anderen Sprache kann dann auch Folgen nach sich ziehen: die Einflüsse auf den Sprachgebrauch und auf den Stil des jeweiligen Autors. Die polnische Muttersprache kann andererseits auch die Produktion der Texte beeinflussen, sie färbt und formt manchmal auch die Zweitsprache. Mit der Wahl einer Sprache oder ihrem Wechsel hängt das Problem der kulturellen Identität und der identitätsstiftenden Funktion der Sprache zusammen. Sprache ist nämlich ein konstituierendes Merkmal für die Identität von nationalen, ethnischen oder kulturellen Gruppen. ${ }^{1}$ Im Hinblick auf die Migrantenliteratur entsteht die Frage, ob die kulturelle Identität durch Sprache bedingt wird und ob der Sprachwechsel einen Identitätswechsel oder doppelte Identität nach sich zieht. ${ }^{2}$ Der Sprachwechsel und die Wahl der Schreibsprache ist ein äußerst wichtiges Problem des individuellen und kollektiven Identitätsverständnisses in der Fremde. Dieter Lamping ist der Meinung, wenn

[...] ein Schriftsteller seine Sprache wechselt und fortan in einer zweiten Sprache oder gar in zwei Sprachen schreibt, bedeutet [dies] nicht weniger, als daß er die - auch von Philologen für natürlich genommene - Bindung eines Werkes an eine Sprache und damit an eine Nationalliteratur aufkündigt. (Lamping 1996: 33)

Die Migrantenschriftsteller, oder nach Trepte „Schriftstellernomaden“, entwerfen ihre Identität neu, aber bei ,einer gleichzeitig beibehaltenen Verwurzelung in der ursprünglichen geokulturellen (sozialen) Landschaft, die (noch) Ursache kultureller Fremdheit ist", woraus sich - im durchaus positiven Sinne - „ein doppeltes Sehen“ und ,hybride kulturelle Mischformen“ ergeben (Trepte 2000: 257). Die Identität kann und muss - im Gegensatz zu den traditionellen Ansätzen - nicht mehr als etwas Statisches, Gleichbleibendes, sondern eher als etwas Dynamisches, Prozesshaftes, Veränderbares verstanden werden.

Radek Knapp, 1964 in Warschau geboren, seit 1994 im deutschsprachigen Raum schriftstellerisch tätig, ${ }^{3}$ wuchs bei den Großeltern in Polen und seit 1976 bei seiner

${ }^{1}$ Georges Lüdi betont, dass der Sprache in den Identitätsprozessen eine Schlüsselrolle zukommt (Lüdi 2007: 43).

${ }^{2}$ Diese Fragen stellt sich Hans-Christian Trepte in seinem Beitrag (vgl. Trepte 2000: 248).

${ }^{3}$ Knapp hat vier Romane (Herrn Kukas Empfehlungen 1999, Der Papiertiger 2003, Reise nach Kalino 2012, Der Gipfeldieb 2015), einen Erzählungsband (Franio 1994), einen essayistischen Reiseführer auf Deutsch geschrieben (Gebrauchsanweisung für Polen 2005) sowie Erzählungen Der Mann, der Luft 
Mutter in Wien auf. ${ }^{4}$ Radek Knapp ist noch im Kindesalter durch die Entscheidung der Erwachsenen in eine deutschsprachige Umgebung versetzt worden und musste die deutsche Sprache in der deutschsprachigen Umgebung bereits im Kindesalter erlernen. Knapp ist durch seinen biografischen Hintergrund eigentlich mit der deutschen Sprache aufgewachsen, so scheint die Wahl seines literarischen Mediums eher eigentlich etwas Selbstverständliches und Natürliches zu sein. Das Deutschlernen war aber für ihn, der am Anfang „nur Polnisch gedacht und geschrieben” habe, wie er behauptet, „wirklich ein Schock” (vgl. Knapp 1996a: 146). Knapp meint: „Deutsch zu lernen ist für einen Polen kein Vergnügen. Noch dazu, wenn dieser zwölfjährig ist. Dann ist Deutsch für ihn nicht nur ein kantiges, raues Kauderwelsch, sondern auch noch die Sprache des Feindes“ (Knapp 2007: 7). ${ }^{5}$ In seinem kurzen Text, der den Titel Kurze Geschichte meiner Sprache trägt, erklärt Radek Knapp seine Entscheidung, literarisch in der deutschen Sprache tätig zu sein:

\footnotetext{
Ich habe in der Fremde eine Sprache gefunden, die mir auf den Leib geschnitten ist. Ich werde sie zwar nie so gut beherrschen wie meine Muttersprache, aber ich werde sie immer dann benutzen, wenn von meinen Gefühlen die Rede sein wird. Sie ist inzwischen nicht nur zu meiner eigenen Sprache geworden, sie ist der rote Faden, der mich zwischen den Kulturen führt, in denen ich lebe. Ich weiß, sie wird mich, und ich werde sie nicht im Stich lassen. (Knapp 1998: 8)
}

Knapp gibt keine präzise Auskunft darüber, warum er ausgerechnet in der deutschen Sprache, und nicht in seiner Muttersprache, seine persönlichen Gefühle und Erlebnisse ausdrücken kann und will. Der Schriftsteller betrachtet auch das Deutsche als seine Arbeitssprache (vgl. Knapp 1996a: 147).

Von den Migrantenautoren, die Deutsch als ihre Literatursprache bewusst gewählt haben und sie in ihrer ästhetischen Funktion gebrauchen, wird Kreativität im Umgang mit Sprache gefordert. Es können dabei hybride Sprachformen entstehen, wenn etwa Elemente sowohl der Herkunftssprache als auch der Sprache der Aufnahmegesellschaft in einem gemischten Sprach-Code verwendet werden. Die Art und Weise der Sprachmischung kann unterschiedlichste Formen annehmen, z. B. können nur einzelne Wortteile, oder Einzelworte aus einer Sprache in die andere eingebaut oder satzweise die

zum Frühstück aß. Erzählung 2017, und Die Stunde der Geburt/The Hour Of Birth. Eine Erzählung zu 41 Grafiken von Alfred Kubin (2017) veröffentlicht. Sein Roman Herrn Kukas Empfehlungen gehört zu den erfolgreichsten Longsellern bei Piper Verlag.

${ }^{4}$ Radek Knapp: „Da musste ich schon eine Woche später in eine fremde Schule, fremdsprachig, total unbekannt. Am Anfang wollte ich gar nicht Deutsch lernen. Das war wirklich ein Schock. Das nächste halbe Jahr wollte ich praktisch weglaufen, zurück nach Polen. Da wurde ich sozusagen aus dem Sattel gerissen, bevor ich noch etwas sagen konnte. Ich muss zugeben: Da war es vorbei mit meiner Jugend!" (vgl. Knapp 1996: 146)

${ }^{5}$ Aus dieser Feststellung Knapps wird noch eine historische Tatsache ersichtlich: Der Gebrauch der deutschen Sprache war im polnischen kollektiven Bewusstsein der Nachkriegszeit durch die negativen Auswirkungen des Zweiten Weltkrieges belastet. Deutsch war die Sprache der Nazis, der Mörder und sein Gebrauch eine gewisse Form des ,nationalen Verrates'. 
Sprache gewechselt werden. Radek Knapp versucht, seine eigene Zweisprachigkeit in die Texte einzubringen, in denen häufig beide Kulturen (die polnische sowie die österreichische/deutschsprachige) und Sprachen koexistieren. Auf der sprachlichen Ebene findet die Vermischung vom Eigenen und Fremden statt (vgl. dazu Lamping 2001: 152). Die Herkunftssprache ist auch im deutschen Text präsent, in der häufigen Verwendung der Termini in der Muttersprache, deutschen Übersetzungen und Übertragungen aus der polnischen Sprache. Obwohl wir in seinem Falle weder mit einer sprachexperimentellen Prosa noch „eine[r] innovative[n] hybride[n] Sprache“ (Esselborn 2009: 46), sondern mit einer traditionellen Erzählweise zu tun haben, in denen die Gesetzmäßigkeiten und Gepflogenheiten des Deutschen berücksichtigt werden, literarisiert der Schriftsteller seine Zweisprachigkeit in seinem Werk und nutzt sprachlich-kommunikative Elemente aus den ihm zur Verfügung stehenden sprachlichen und kulturellen Systemen, überschreitet die Sprachgrenzen und verwendet in seinen Texten auch polnischsprachige Elemente im deutschsprachigen Kontext. Auf diese Weise wird die polnische Sprache als Element der Konstruierung seiner Texte angesehen und zwei Kulturen werden in einem Text nicht nur auf der thematischen (inhaltlichen) Ebene, sondern auch in der sprachlichen Gestaltung in Verbindung gebracht.

Knapp übernimmt zum Beispiel in seiner Rede oft Elemente aus der polnischen Sprache und wechselt nicht selten den Kode (vgl. Pütz 1994: 137), wobei der Übergang von einer Sprache zur anderen „fließend“ erfolgt. Er verwendet aus inhaltlichen oder sprachlich-ästhetischen Gründen in seinen deutschsprachigen literarischen Texten viele polnischsprachige Elemente, die nicht nur als sprachliche Gestaltungsmittel der Texte, „kommunikativ auffällige, signalhaft wirkende Mittel“ (Fix, Poethe \& Yos 2001: 51), sondern auch als mögliche Trägertypen eines inter- oder vielleicht sogar transkulturellen Potentials betrachtet werden können. Die Manifestationen von Sprachenmischung, der Gebrauch der beiden Sprachen, bzw. die Heranziehung der polnischen Sprache können möglicherweise als Ausdruck sowohl seiner doppelten identitären Verortung als auch seines Widerstandes gegen die völlige Assimilierung betrachtet werden (vgl. Gugenberger 2011: 24). Die Manifestation von Zweisprachigkeit kann auch als Stilmittel zur Veranschaulichung der Position von Schriftstellern zwischen zwei Kulturen interpretiert werden (vgl. Berkenbusch, Heinemann 1995).

Die polnische Sprache signalisiert außerdem auch die kulturellen Konstellationen, u. a. die kulturelle Herkunft der Protagonisten Knapps oder ihre Zugehörigkeit zur polnischen Sprach- und Kulturgemeinschaft, die „Raum-Zeit-Konstellation, in der das Werk zum Teil angesiedelt ist“ (Chiellino 2002: 43), sie verleiht der Figurenrede (und den Texten) Authentizität. Fremdsprachige Einschübe werden in die Texte bewusst integriert, um beabsichtigte Stileffekte zu erreichen oder spezifische Konnotationen beim Leser auszulösen. Durch den Gebrauch von Sprachelementen aus der eigenen Herkunftssprache spielt der Schriftsteller mit dem Effekt des fremden Wortes und versucht auf diese Weise auch, seine eigene Zweisprachigkeit in die Texte einzubringen. Die eingeschobenen polnischsprachigen Elemente werden aus inhaltlichen Gründen 
verwendet, sie sollen auch darauf aufmerksam machen, dass sich die Handlung des jeweiligen Werkes entweder in einer polnischsprachigen Umgebung abspielt oder dass die Figuren polnischer Herkunft sind. Einerseits dienen sie der Darstellung der Authentizität und schaffen eine Atmosphäre der kulturellen Vertrautheit, andererseits aber betonen sie auch die Andersheit und Fremdheit der beschriebenen Welt. Die Sprache wird somit zum Ort eines möglichen Dialogs.

Das literarische Reiseessay Gebrauchsanweisung für Polen nimmt in dieser Hinsicht eine Sonderposition ein, weil es auf eine informative Weise ein vielfältiges Bild von Polen, polnischer Kultur und Geschichte sowie von polnischen Städten, Landschaften und Realien vermittelt. Die polnischsprachigen Einschübe fungieren als Ausdrucksmittel der kulturellen Vielfalt und sind maßgeblich für den Kulturtransfer. Für ihre Dekodierung ist ein gewisses Vorwissen des Lesepublikums gefragt. So kommen polnische Eigennamen, in denen sich die Sprachenkontakte in den Texten manifestieren, ausgesprochen häufig vor: Personennamen der Figuren oder Namen von historischen Personen, geographische Namen, wie Namen von Landschaften, Orten, Straßen, Namen von Institutionen und Organisationen, Produkt- und Markennamen, Namen von materiellen und immateriellen Kulturgütern, wie Namen von historischen Ereignissen, Bauten und Kunstwerken, Namen von Liedern, Speisen, Titel von Büchern und Filmen. Sie sind meistens mit bestimmten Konnotationen und Assoziationen verbunden. Dabei wird häufig auf (kultur-)spezifisches Wissen verwiesen oder dieses wird vorausgesetzt. Im Folgenden sollen einige Beispiele genannt werden.

Es werden von Knapp polnischsprachige Personennamen und auch inoffizielle Personenbenennungen (wie Kose-, Spitz, Neck- und Scherznamen) verwendet, die als Manifestationen/Träger der polnischen Kultur betrachtet werden können (Auswahl): Nowak, Bogumil Trombka, Koralik, Malinka, Miodek, Maniek, Smolny, Franio, Antoni, Wacek, Janek, Maj und Majowa, Krysia, Monika, Sosia, Kamila, Kasia, Lusia, Sawka, Pasur, Mostek, Hanka, Jola, Waldemar, Bolek, Ala, Ludwik, Marcin. Die originelle polnische Schreibweise der Namen, die zum „kulturellpolnischen“ Kolorit der Erzählungen beitragen, wird von Knapp beibehalten, es gibt nur wenige, die von dem Autor „eingedeutscht“ worden sind (Trombka, Felix, Julius, Sosia, Frantischek, Muschek, Motill, Pasur). Oftmals tauchen in Knapps Texten Namen geographischer (Landschaften, Orte, Straßen) oder historischer (Namen von historischen Ereignissen, Bauten und Kunstwerken, Namen von Liedern, Speisen, Titel von Büchern, Zeitschriften, Filmen) Realia auf, z. B.: Sopot, Miedzyzdroje (Knapp 2005: 54), Wieliczka (Knapp 2005: 148), Rysy, Jaskinia Śnieżna (Knapp 2005: 53), „Radio Maria” (Knapp 2005: 15), „Gazeta Wyborcza“ (Knapp 2005: 117f), „Polityka” (Knapp 2005: 117), ,Wprost” (Knapp 2005: 117), „Czterej pancerni i pies“ (Knapp 2005: 122), „Kapitan Klos” (Knapp 2005: 122), „Potop”, „Janosik” (Knapp 2005: 122), „Solidarność-Bewegung” (Knapp 2005: 64, 69), Legende über Wars und Sawa (Knapp 2005: 34), „Bigos“ (Knapp 2005: 16), „,barszcz ukraiński [...] ruskie pierogi” (Knapp 2005: 86), „kiełbasa zwyczajna [...] oder myśliwska” (Knapp 2005: 87), „miod pitny” (Knapp 2005: 90), „Wyborowa” (Knapp 2005: 90). 
Die polnischsprachigen Einschübe werden in den meisten Texten zur Wiedergabe der polnischen Laute mit diakritischen Zeichen versehen (obwohl nicht immer konsequent, vgl. Knapps Gebrauchsanweisung für Polen). In vielen Texten werden sie außerdem typographisch (mit Kursivdruck) hervorgehoben. Die Kursivsetzung hat zum Ziel, den Leser auf das fremdsprachige Element aufmerksam zu machen und fungiert auch als „visueller Marker der Fremdheit von Lexemen oder komplexen Ausdrücken“ (Gymnich 2007: 74). Die polnischsprachigen Passagen oder Elemente werden, wie schon erwähnt, in der Regel in den Texten entweder durch Erläuterungen eingeleitet oder ins Deutsche übersetzt, wovon die folgenden ausgewählten Beispiele zeugen: „beim Landesnamen: Polska” (Knapp 2005: 93 u. 94), ,dzien dobry [...], was soviel wie guten Tag bedeutet [...].“ (Knapp 2005: 24), „Chrząszcz brzmi w trzcinie (bitte aussprechen: Chschonschtsch bschmi w tschtzinje), was frei übersetzt heißt: Ein Käfer zirpt im Schilf” (Knapp 2005: 27f), „Das Wort Niemcy kommt von niemy, was soviel wie stumm bedeutet” (Knapp 2005: 29), „Polska to wspaniaty kraj (Polen ist ein wunderbares Land)” (Knapp 2005: 31), „Der Name Wałęsa kommt von watęsać, was soviel heißt wie »sich herumtreiben « oder »herumschlawinern«" (Knapp 2005: 64), „eine Scheinwährung, Bony towarowe [...]” (Knapp 2005: 72), „die Kuttelfleckensuppe (flaki)” (Knapp 2005: 86), „Ukrainischer Borschtsch (barszcz ukraiński) und Russische Piroggen (ruskie pierogi)” (Knapp 2005: 86), „kietbasa zwyczajna, frei übersetzt, »gewöhnliche Wurst«" (Knapp 2005: 87), „miod pitny, ein alkoholisches Honiggetränk” (Knapp 2005: 90), „Aus dem Programm des Kabarettklassikers Marcin Wolski” (Knapp 2005: 76), „der polnische Wissenschaftler Professor Miodek“ (Knapp 2005: 30), „der charismatische Priester Jerzy Popiełuszko, der wegen seiner oppositionellen Arbeit im Jahr 1984 ermordet wurde“ (Knapp 2005: 82), „Der orthodoxe Geistliche Rydzyk” (Knapp 2005: 84), „Die berühmteste Kriegsserie Polens hieß »Czterej pancerni i pies« was so viel wie: »Vier Panzersoldaten und ein Hund« bedeutete.” (Knapp 2005: 122), ,»Pan Samochodzik i templariusze«. Übersetzt: »Pan Samochodzik und die Tempelritter«. [...] Pan Samochodzik, was frei übersetzt »Herr kleines Auto« bedeutet" (Knapp 2005: 131f.).

Wie schon erwähnt, ist zu beachten, dass der größte Teil des polnischsprachigen Wortschatzes entweder durch deutsche Erläuterungen eingeleitet oder ins Deutsche übersetzt und somit in den sonst deutschsprachigen Text integriert wird. Auf diese Weise lassen sich diese Wörter und Sätze auf Polnisch vom deutschsprachigen Leser ohne Polnischkenntnisse entschlüsseln und nachvollziehen. Die polnischen Formulierungen blieben in den Texten nur relativ selten unübersetzt, weil der deutschsprachige Rezipient durch das Ausbleiben einer Übersetzung oder Erklärung mit einem Alteritätsgefühl konfrontiert wird, wenn ihm die Bedeutung der polnischsprachigen Einschübe verborgen bleibt (Gymnich 2007: 61). Knapp bedient sich der deutschen Sprache souverän, weil er das Deutsche als Sozialisationssprache erlernt hat. Sprachlich auffallend ist - neben der sprachlichen Hybridisierung - die häufige Verwendung der Umgangssprache sowie der gesprochenen Alltagssprache, die manchmal sehr direkte, 
unvermittelte Ausdrucksweise, wodurch seine Texte einen hohen Grad an Lebendigkeit erreichen. Das Deutsche und das Polnische werden miteinander in Verbindung gesetzt.

Die Wahl einer bestimmten Sprache kann auch als eine Strategie der Identitätskonstruktion gesehen werden, in der jemand kulturelle und sprachliche Präferenzen ausdrückt und sich dadurch selbst kategorisiert. Der Migrantenschriftsteller positioniert sich im literarischen Raum und will seine Zugehörigkeit markieren. Der Gebrauch der deutschen Sprache ist im Falle Knapps auch in der Möglichkeit begründet, in Westeuropa literarisch tätig zu sein und ein größeres Lesepublikum anzusprechen. ${ }^{6}$ Das Deutsche als literarisches Ausdrucks- und Publikationsmedium begründet keine homogenen nationalen Identitäten, sondern erfüllt die Funktion eines Transitraumes, einer-zone (vgl. Adam, Hahn, Puchalski \& Światłowska 2007b), eines transkulturellen Raumes der gegenseitigen Durchdringung und Verflechtung des Kulturellen sowie der Transformation nationaler Zugehörigkeiten und der kulturellen Identität. Der Schriftsteller Knapp versucht, seine eigene Zweisprachigkeit in die Texte einzubringen, in denen häufig beide Kulturen und Sprachen koexistieren. Für den Schriftsteller als „Brücken-Menschen“ (vgl. Schoen 1996: 25) öffnet sich in seiner deutschen Literatursprache ein produktiver Zwischenraum, ein Kreuzungspunkt der Ordnungen und Systeme, der nicht vom Entweder-oder, sondern von der Denkfigur des Sowohl-als-auch geprägt ist, selbst dann, wenn wir mit keiner sprachexperimentellen Prosa und einer innovativen Sprache - wie im Falle Knapps - zu tun haben.

\section{Literatur}

Adam, J., Hahn, H.-J., Puchalski, L. \& Światłowska, I. (Hrsg.) (2007). Transitraum Deutsch. Literatur und Kultur im transnationalen Zeitalter. Wrocław, Dresden: Neisse.

Berkenbusch, G. \& Heinemann, U. (1995). El amante bilingüe - Interkulturelle Konfliktivität, Verfahren ihrer Versprachlichung im zeitgenössischen spanischen Roman und das Problem einer angemessenen Übersetzung. LiLi. Zeitschrift für Literaturwissenschaft und Linguistik, 97, 48-72.

Chiellino, C. (2002). Der interkulturelle Roman. In A. Blioumi (Hrsg.), Migration und Interkulturalität in neueren literarischen Texten (S. 41-54) München: iudicium.

Esselborn, K. (2009). Neue Zugänge zur inter/transkulturellen deutschsprachigen Literatur. In H. Schmitz (Hrsg.), Von der nationalen zur internationalen Literatur. Transkulturelle deutschsprachige Literatur und Kultur im Zeitalter globaler Migration (S. 43-58). Amsterdam: Rodopi.

Fix, U., Poethe, H. \& Yos, G. (2001). Textlinguistik und Stilistik für Einsteiger. Ein Lehr- und Arbeitsbuch. Unter Mitarbeit von R. Geier. Frankfurt a. M.: Peter Lang.

Gugenberger, E. (2011). Hybridität und Translingualität: lateinamerikanische Sprachen im Wandel. In E. Gugenberger \& K. Sartingen (Hrsg.), Hybridität - Transkulturalität - Kreolisierung. Innovation und Wandel in Kultur, Sprache und Literatur Lateinamerikas (S. 11-49). Berlin: LIT.

\footnotetext{
${ }^{6}$ „Es ist ziemlich wichtig, dass man eine Weltsprache beherrscht, wenn man schreiben will. Wie ein Wissenschaftler [...] muss ich Bücher von Autoren, die mich interessieren, lesen, wenn sie gerade erschienen sind. Das ist im Polnischen unmöglich, auch heute noch“, meinte Radek Knapp in einem Interview aus dem Jahr 1996 (vgl. Knapp 1996a: 147f.).
} 
Gymnich, M. (2007). Metasprachliche Reflexionen und sprachliche Gestaltungsmittel im englischsprachigen und postkolonialen und interkulturellen Roman. Trier: Wissenschaftlicher Verlag.

Knapp, R. (1996a). ,Wien und Warschau sind für mich zu einer Stadt zusammengeschmolzen “. Radek Knapp im Gespräch mit Christa Stippinger. In Ch. Stippinger (Hrsg.), Jeder ist anderswo ein Fremder. Interkulturelle Reihe des Vereins Exil im Amerlinghaus (S. 145-148). Wien: edition exil.

Knapp, R. (1996). Franio. Erzählungen. Reinbek bei Hamburg: Rowohlt.

Knapp, R. (1998). Kurze Geschichte meiner Sprache. In Ch. Stippinger (Hrsg.), Weltenzwischenwelten (S. 7-8). Wien: edition exil.

Knapp, R. (1999). Herrn Kukas Empfehlungen. Roman. München: Piper.

Knapp, R. (2005). Gebrauchsanweisung für Polen. München: Piper.

Knapp, R. (2007). Mein erstes Schragl. In Ch. Stippinger (Hrsg.), Best of 10. Anthologie. 10 Jahre Exil-Literaturpreise „Schreiben zwischen den Kulturen“ 1997-2006 (S. 7). Wien: edition exil.

Lamping, D. (1996). Haben Schriftsteller nur eine Sprache? Über den Sprachwechsel in der Exilliteratur. In D. Lamping (Hrsg.), Literatur und Theorie: Über poetologische Probleme der Moderne (S. 33-48). Göttingen: Vandenhoeck \& Ruprecht.

Lamping, D. (2001). Über Grenzen. Eine literarische Topographie. Göttingen: Vandenhoeck \& Ruprecht. Linhartová, V. (1997). For an Ontology of Exile. In Literature, Prison, Exile. Readers International, Praha: Nadace Readers International (S. 152-162). České centrum Mezinárodního PEN klubu.

Lüdi, G. (2007). ,, Mehrsprachige Repertoires und plurielle Identität von Migranten: Chancen und Probleme“. In I. De Florio-Hansen \& A. Hu (Hrsg.), Plurilingualität und Identität. Zur Selbst- und Fremdwahrnehmung mehrsprachiger Menschen (S. 39-58).Tübingen: Stauffenburg.

Pütz, M. (1994). Sprachökologie und Sprachwechsel. Die deutsch-australische Sprechergemeinschaft in Canberra. Frankfurt a. M.: Peter Lang.

Schoen, U. (1996). Bi-Identität, Zweisprachigkeit, Bi-Religiosität, doppelte Stadtbürgerschaft. Zürich, Düsseldorf: Walter.

Stanišić, S. (2008). Wie ihr uns seht. Über drei Mythen vom Schreiben der Migranten. In U. Pörksen $\&$ B. Busch (Hrsg.), Eingezogen in die Sprache, angekommen in der Literatur. Positionen des Schreibens in unserem Einwanderungsland (S. 104-109). Göttingen: Wallstein.

Trepte, H.-Ch. (2000). Polnische Exilliteratur - Sprache und Identität. In Ł. Gałecki \& B. Kerski (Hrsg.), Die polnische Emigration und Europa 1946-1990. Eine Bilanz des politischen Denkens und der Literatur Polens im Exil (S. 247-264). Osnabrück: fibre.

Trepte, H.-Ch. (2013). W poszukiwaniu innej rzeczywistości. In E. Teodorowicz-Hellmann \& J. Gesche (Hrsg.), Między językami, kulturami, literaturami. Polska literatura (e)migracyjna w Berlinie i Sztokholmie po roku 1981, 81-97. Stockholm Slavic Papers, no. 22. Stockholm: Stockholms universitet Slaviska institutionen. 